\title{
Idiopathic Bilateral Strio-Pallido-Dentate Calcinosis (Fahr's Disease) and Chronic Subdural Haematoma In A 40 Year Old Woman with Depression: A Case Report
}

\author{
Danfulani Mohammed ${ }^{1}$, Gele H. Ibrahim ${ }^{1}$, Ma'aji M. Sadisu', Saidu A. Sule ${ }^{1}$, \\ Muhammad A. Musa ${ }^{2}$ \\ ${ }^{\text {I}}$ (Department of Radiology, Faculty of Clinical Sciences, College of Health Sciences, Usmanu Danfodiyo \\ University, Sokoto, Nigeria). \\ ${ }^{2}$ (Department of Anatomy, Faculty of Basic Medical Sciences, College of Health Sciences, Usmanu Danfodiyo \\ University, Sokoto, Nigeria).
}

\begin{abstract}
Idiopathic bilateral strio-pallido-dentate calcinosis is a rare disorder characterized by symmetrical calcifications noticeable in parts of the brain. We present the case of idiopathic bilateral strio-pallido-dentate calcinosis (Fahr's disease) in a 40 year old woman with depressive symptoms. Computed Tomography scan of the brain showed extensive bilateral symmetrical calcifications in the basal ganglia and cerebellum as well as chronic left fronto-parietal subdural haematoma.
\end{abstract}

Keywords: Fahr's disease, woman, calcification, haematoma.

\section{Introduction}

Idiopathic bilateral strio-pallido-dentate calcinosis also known as Fahr's disease is a rare disorder characterized by symmetrical calcifications in basal ganglia, thalamus, cerebellar dentate nucleus and cerebral white matter [1]. It was first described by a German pathologist-Fahr in 1930 [2]. Bilateral symmetrical intracranial calcifications of the strio-pallido-dentate system are detectable in $0.7-1.2 \%$ of computed tomographic scans and may be caused by different disease conditions that includes idiopathic, familial or calcium and parathyroid hormone metabolism disorders (Fahr's syndrome) [1,2].

The aetiology of Fahr's disease is unknown. Meanwhile clinically these patients show developmental and motor disability, tetany, paraesthesia, cataract and epileptic syncope [3]. The diagnosis is based on clinical features, neuroimaging and exclusion of other primary causes [4]. The rarity of this condition in this environment and unusual presentation prompted the report of this case.

\section{Case Report}

We present a 40 year old female, a civil servant who presented to the Psychiatric Unit of Usmanu Danfodiyo University Teaching Hospital Sokoto with one year history of poor sleep and apetite, headache and crying for no reason. No history of recent family upset or loss of member of the family. No fever or history of seizures. She had a road traffic accident 13 years ago with scalp laceration but there was no loss of consciousness. She has 3 children, 2 male and one female who are all alive. No family history of depression or any psychiatric illness.

Examination showed a middle aged woman who was mute, afebrile, anicteric and not pale. Musculoskeletal and cardiovascular system examinations were normal. Blood pressure was $110 / 80 \mathrm{mmHg}$. An assessment of moderate depression was made. Laboratory investigations showed normal renal function. The serum calcium, Phosphate, Parathyroid hormone, thyroid hormone and blood sugar were all normal. She was subsequently placed on antidepressants. She had electroconvulsive therapy (ECT) on three occasions with minimal improvement of symptoms. She was then referred to Radiology department for cranial computed tomographic (CT) scan to rule out intracranial lesion.

The CT scan showed extensive bilateral symmetrical calcific densities $(\mathrm{HU}=123)$ in the basal ganglia involving the head of the caudate nucleus, putamen, globus pallidus, the thalamus and the dentate nucleus of the cerebellum (Figures 1 and 2). There was also a concavo-convex area of hypodensity in the left fronto-parietal region adjacent to the cranial vault, with associated midline shift to the right (Figure 1). No fracture was demonstrated. An assessment of Fahr's disease with chronic subdural haematoma was made. She had burr hole with drainage of the subdural haematoma and continued the antidepressant therapy. There was remarkable improvement of symptoms and she was discharged and is on regular follow up visits. 


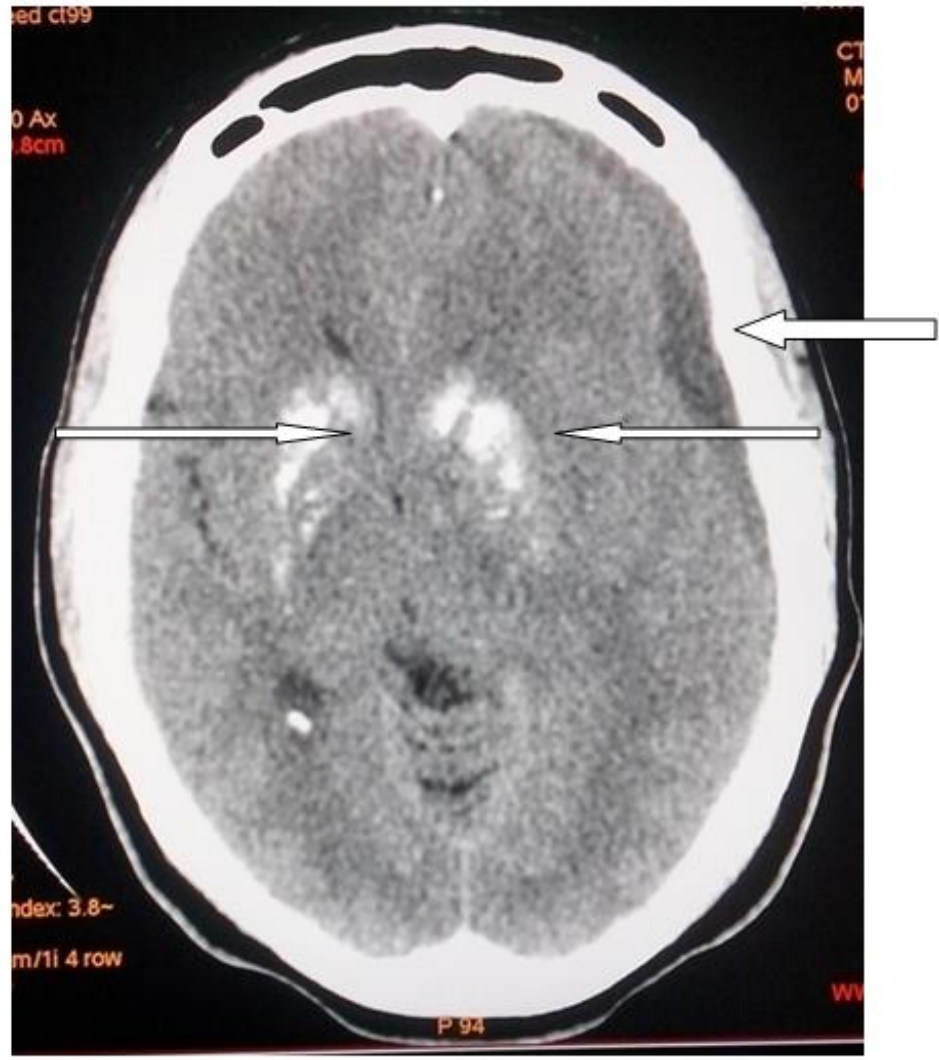

FIGURE 1: Non contrast computed tomogram of the brain showing bilateral symmetrical calcific densities in the basal ganglia and thalamus (thin arrows). There is a concavo-convex hypodensity in the left fronto-parietal region due to chronic subdural haematoma (thick arrow) with associated midline shift to the right and compression of both frontal horns of the lateral ventricle more marked on the left side.

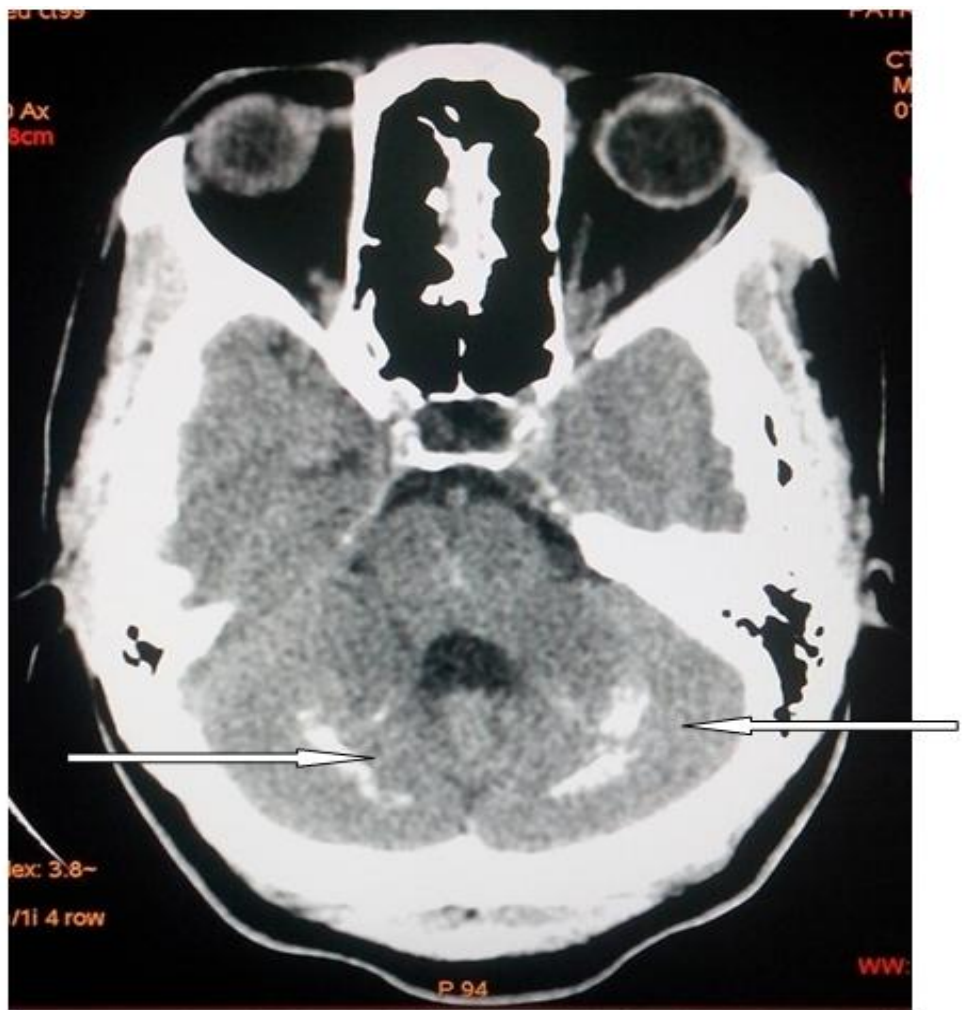

FIGURE 2:Non contrast computed tomogram of the brain at the level of the cerebellum showing bilateral symmetrical calcific densities in the cerebellum (arrows). 


\section{Discussion}

Idiopathic bilateral strio-pallido-dentate calcinosis (Fahr's disease) is characterized by sporadic or familial idiopathic calcifications of the basal ganglia, dentate nucleus of the cerebellum and centrum semiovale. Typical age of presentation is in middle age between the $4^{\text {th }}$ and $6^{\text {th }}$ decades of life (as seen in this case), although an early onset type may also occur [4]. Early onset types show a more progressive deteriorating course with features mimicking schizophrenia and resulting in presenile dementia $[4,5,6]$.

People with Fahr's disease frequently present with movement disorders such as rigidity, hypokinesia, tremor, choreosthetosis and ataxia, with frontal subcortical patterns of behavioral disturbances such as psychosis, mood disorders and dysexecutive neuropsychological syndrome. Other neurological features are seizures or stroke-like events [6]. Abubakar and Saidu, [2] reported a case of idiopathic bilateral strio-pallidodentate calcinosis in Sokoto who presented with hemichorea [2]. In a similar case, Calabro et al., [6] reported Fahr's disease presenting with dementia, behavioral and mood disorders [6].

Patients with Fahr's disease who present with predominant frontal lobe syndrome and dementia show a massive reduction in glucose metabolism in both the basal ganglia and frontal lobes which may correlate with personality change in the patient. The involvement of frontal subcortical circuits provides a hypothetical framework for the interpretation of cognitive and psychotic problems in Fahr's disease $[4,7,8]$.

The diagnosis of Fahr's disease relies on the combination of clinical and radiological features as well as exclusion of other causes of intracranial calcifications [2,4]. On imaging, intracranial calcifications can be demonstrated on plain skull radiograph, Computed tomography (CT) and magnetic resonance imaging (MRI). However CT is considered more sensitive than skull radiograph and MRI for demonstrating calcified deposits in Fahr's disease. Skull radiograph may not always reveal calcifications and if present the exact location of the calcification may not be delineated. On MRI, calcifications appear as area of signal void or the findings may be non-specific. It has been suggested that the unusual hyperintense $\mathrm{T}_{2}$ weighted images seen on MRI may reflect a slowly progressive metabolic or inflammatory process in the brain, which subsequently calcifies. When calcific deposits seen on CT present as hyperintensities in $\mathrm{T}_{1}$ weighted images, this might be due to the paramagnetic effect of the different mineral ions present in strio-pallido-dentate deposits, mainly manganese or due to proteins and mucopolysaccharides binding the mineral ions [3].

Computed tomography in Fahr's disease reveals extensive bilateral and symmetrical calcific hyperdensities in the caudate nucleus, lentiform nucleus, thalamus and dentate nucleus as demonstrated in the case here presented (Figures 1 and 2). Hyperdensities may also be demonstrated in the subcortical white matter [3]. This was not seen in this case. Computed tomographic findings of intracranial calcifications in Fahr's disease can be misinterpreted as intracranial haemorrhage in an emergency setting [1]. Kara et al., [1] reported a case of Fahr's disease accompanied by epidural haematoma in a patient with head trauma [1]. The index case also had chronic subdural haematoma (Figure 1). The cause of the haematoma in the index patient is not known, but could be due to a head trauma sustained unnoticed during repeated electroconvulsive therapy.

The differential diagnosis of Fahr's disease includes causes of basal ganglia calcifications such as old age, hypoparathyroidism, birth anoxia, radiation, lead and carbon monoxide poisoning or infections such as retroviral infection, toxoplasmosis and cytomegaloviral infection. In old age, birth anoxia and toxin-induced injuries, calcifications are generally confined to the globus pallidus [9] in contrast with this patient who had calcifications outside the globus pallidus. The calcifications in infectious disease are usually asymmetric and not restricted to the basal ganglia. The calcifications of Fahr's disease are not distinguishable from those secondary to hypoparathyroidism on imaging. Therefore, there is need for biochemical or hormonal investigations [9].

The treatment of Fahr's disease is symptomatic. However, antipsychotics may be indicated in those with psychotic symptoms and behavioural problems $[4,8]$. The index patient presented with depressive symptoms and was treated with antidepressant. She also had drainage of the subdural haematoma found on CT scan. Since there is no known cure or specific treatment currently and coupled with the fact that the appearance of calcification is age-dependent, Fahr's disease may progress.

\section{Conclusion}

A case of idiopathic bilateral strio-pallido-dentate calcinosis (Fahr's disease) in a 40 year old woman with depressive symptoms is presented. She had brain CT which showed extensive bilateral symmetrical calcifications in the basal ganglia and cerebellum as well as chronic left fronto-parietal subdural haematoma. She had drainage of the haematoma and improved on antidepressants.

\section{References}

[1] H. Kara, A. Bayir, A. Ahmet, M. Akinci, S. Degirmenci and H. Alp. Fahr's disease accompanied by epidural haematoma: a case report. Case Study Case Rep., 3(4), 2013, 138-143.

[2] S.A. Abubakar and S. Saidu. Idiopathic bilateral strio-pallido-dentate calcinosis (Fahr's disease): A case report and review of the literature. Ann Afr Med., 11, 2012, 234-7.

[3] O.P. Sharma, S. Senthil. Fahr's disease: Report of two cases. Asian J Med Sci., 2, 2011, 120-123. 
Idiopathic Bilateral Strio-Pallido-Dentate Calcinosis (Fahr's Disease) and Chronic Subdural ..

[4] A. Mittal, B.K. Agrawal, A. Mittal, G. Praveen, A. Jain. Fahr's syndrome: A rare case of idiopathic basal ganglia calcifications. $J$ Ind Acad Clinic Med., 11(3), 2010, 239-41.

[5] P.J. Modrego, J. Mojenoro, M. Serrano and N. Fayed. Fahr's syndrome presenting with pure and progressive presenile dementia. Neurol Sci.,26(5), 2005, 367-9.

[6] R.S. Calabro, L. Spadaro, A. Marra and P. Bramanti. Fahr's disease presenting with dementia at onset: A case report and literature review. Behavioural neurol., 2014, 1-3.

[7] D.C. Weisman, R. Yaari, L.A. Hansen and L.J. Thal. Density of the brain, decline of the mind: an atypical case of Fahr's disease. Arch Neurol., 64(5), 2007, 756-757.

[8] D. Ghormode, U. Maheshwari, N. Kate and S. Grover. Fahr's Disease and Psychiatric Syndromes: A Case Series. Ind. Psychiatry J., 20(2), 2011, 136-138.

[9] B. Menon and C.V. Harinarayan. Similar calcifications of the brain on computed tomography, but different etiologies. Ann Ind Acad neurol., 12(2), 2009, 134-135. 